\title{
Intrinsic structure perspective for MIPS interfaces in two dimensional systems of Active Brownian Particles
}

\author{
Enrique Chacon \\ Instituto de Ciencia de Materiales de Madrid, CSIC, 28049 Madrid, Spain \\ Francisco Alarcón \\ Departamento de Estructura de la Materia, Física Térmica y Electrónica, \\ Facultad de Ciencias Físicas, Universidad Complutense de Madrid, 28040, Madrid, Spain and \\ Departamento de Ingeniería Física, División de Ciencias e Ingenierías, \\ Universidad de Guanajuato, Loma del Bosque 103, 37150 León, Mexico. \\ Jorge Ramírez \\ Dep. de Ingeniería Química, ETSI Industriales, \\ Universidad Politécnica de Madrid, 28006 Madrid, Spain. \\ Pedro Tarazona \\ Departamento de Fúsica Teórica de la Materia Condensada, \\ Condensed Matter Physics Center (IFIMAC), Universidad Autonoma de Madrid, 28049 Madrid, Spain \\ Chantal Valeriani \\ Departamento de Estructura de la Materia, Física Térmica y Electrónica, \\ Facultad de Ciencias Físicas, Universidad Complutense de Madrid, 28040, Madrid, Spain
}

(Dated: February 7, 2022)

Suspensions of Active Brownian Particles (ABP) undergo motility induced phase separation (MIPS) over a wide range of mean density and activity strength 1], which implies the spontaneous aggregation of particles due to the persistence of their direction of motion, even in the absence of an explicit attraction. Both similarities and qualitative differences have been obtained when the MIPS is analysed in the same terms as a liquid-gas phase coexistence in an equilibrium attractive system. Negative values of the mechanical surface tension have been reported, from the total forces across the interface, while the stable fluctuations of the interfacial line would be interpreted as a positive capillary surface tension [2], while in equilibrium liquid surfaces these two magnitudes are equal. We present here the analysis of 2D-ABP interfaces in terms of the intrinsic density and force profiles, calculated with the particle distance to the instantaneous interfacial line. Our results provide a new insight in the origin of the MIPS from the local rectification of the random active force on the particles near the interface. As it had been pointed, that effect acts as an external potential [3] that produces a pressure gradient across the interface, so that the mechanical surface tension of the MIPS cannot be described as that of equilibrium coexisting phases; but our analysis shows that most of that effect comes from the tightly caged particles at the dense (inner) side of the MIPS interface, rather than from the free moving particles at the outer side that collide with the dense cluster. Moreover, a clear correlation appears between the decay of the hexatic order parameter at the dense slab and the end of the MIPS as the strength of the active force is lowered. We test that with the strong active forces required for MIPS, the interfacial structure and properties are very similar for ABP with purely repulsive (WCA-LJ model truncated at its minimum) and when the interaction includes a range of the LJ attractive force.

\section{INTRODUCTION}

Active matter focuses on systems composed of particles capable of consuming energy in order to move, constantly driving themselves away from equilibrium [4]. Characteristic features of a bulk suspension of active particles are collective behaviours such as large scale directed motion, dynamic clusters formation or non-equilibrium phase separation. A numerical model that has been thoroughly exploited to understand such properties consists of a suspension of Active Brownian Particles (ABP). ABP are self-propelled Brownian particles whose inter-particle interactions can be either purely repulsive [1, 5] or contain also some range of attractive force 6 [8].

Depending on the nature of inter-particle interactions and of the particles' activity, the system forms different steady state structures. On the one side, a dilute suspension of attractive ABP is characterised by a distribution of dynamic clusters whenever the inter-particle attraction strength is enough to compete with the particles' activity 6 - 9], without any phase separation taking place. Clustering is due to a balance between new particles joining already formed clusters and large clusters breaking into smaller ones $10-12]$. On the other side, a suspension of repulsive ABP undergoes motility induced phase separation (MIPS) for relatively high densities and 
activity strength [1, 13]. MIPS implies a spontaneous aggregation of particles due to the persistence of their direction of motion, even in the absence of an explicit attraction. MIPS has been detected in experimental setups of active colloids [11, 14 -16] and thoroughly characterised in numerical simulations of Active Brownian particles (ABP) [1, 5, 17 23]. Moreover, MIPS cannot be avoided in the presence of inertial effects [24, 25], when considering a suspension of active particles moving on a lattice [26, 27], or when an amount of passive particles is added to the active suspension [28 30].

Trying to understand this non-equilibrium phase separation via a mechanical equation of state, one could draw similarities with the interfacial properties of equilibrium phases. However, a clear agreement has not been reached on the physical properties such as the surface tension [3, 31 35]. The main reason behind the disagreement can be summarised in the difference between considering activity giving rise to a spatially uniform stress (the swim pressure [36 38]) or activity giving rise to a spatially varying body force 3 , 39].

Even though the swim pressure has been successfully used to determine the onset of MIPS [21, 40 45], when dealing with localised phenomena, adding the swim pressure to the total pressure results in a negative surface tension, that in equilibrium phase coexistence would imply an unstable interface. Considering a "stable" (even though largely fluctuating) dense-dilute MIPS slab configurations in a non-square box [2, 31], it has been shown that 2] the pressure formulation, combining the swim pressure with the Kirkwood and Buff formulation for the surface tension, leads to a negative mechanical interfacial tension compatible with a positive surface stiffness, or capillary surface tension. This is a strongly nonequilibrium effect, since from coexisting phases in a passive attractive system the mechanical and capillary surface tensions are equal [46].

To reconcile a stable interface with negative values of surface tension, the authors of Ref.32] discovered a strong correlation between the local curvature of the fluctuating interface and the magnitude of the surface tension. The local curvature is responsible for a local tangential motion of particles within a surface layer both in the dense and dilute phase: the combined particles' motion in the dense and dilute phase leads to a stiffening interface that reduces the amount of local fluctuations.

Another way to reconcile a stable interface with a negative values of the surface tension consists in not including the swim pressure in the stress calculations, as in Ref. [2]. Instead, one can consider the effect of particle's activity via a body force, generated by the net alignment of $\mathrm{ABP}$ at the interface [3]. As a result, this approach leads to a negligible surface tension in the dense-dilute interface of phase separated ABP [47]. The crucial difference with the other perspective is that, since the activity of the particles creates a net mean force at the interfaces, the two MIPS phases are like equilibrium regions with different potential energies, and therefore different bulk pressures, rather than being similar to coexisting phases at equilibrium. The mechanical surface tension across a (smooth) external potential step, that separates regions with different pressure, may be negative in equilibrium systems, and it certainly differs from the (always positive) capillary surface tension extracted from the fluctuations of that (externally induced) interface.

Therefore, a key point to understand the physics of MIPS is the origin and structure of that self-sustained effective external potential formed at the interfaces. A major issue present when extracting information on that effect from ABP simulations is that, at any fixed point $(x, y)$ located on the interfacial region, the strong interfacial fluctuations average both density and force contributions of instantaneous configurations: this affects the point's local environments, mixing low density and high density contributions, from both sides of the instantaneous boundary of the dense cluster. The same problem appears in molecular dynamics simulations of equilibrium liquid surfaces, giving rise to density profiles that become smoother for larger sizes, without a thermodynamic limit.

The Capillary Wave Theory (CWT) [48, 49] formalizes the account of these interfacial fluctuations and, although some of its hypothesis (like the equivalence between the macroscopic and the capillary surface tensions) become problematic for active systems away from equilibrium [3, 33], it has already been used as a generic mesoscopic framework to describe the fluctuating MIPS interfaces 32. In particular, we take advantage of the methods developed to establish a quantitative link between the CWT description of the interfaces as smooth fluctuations shapes, and the particle positions in computer simulations [50? , 51], both for equilibrium and dynamical properties.

In the present work, we extend that analysis, with emphasis on obtaining intrinsic profiles for the density and for the active force acting on the particles. These intrinsic profiles give a sharper view for the formation of MIPS, with the focus on the inner side of the interface, where the particles are already quite tightly caged by their neighbours in local hexatic order structures. The decay of the capillary tension towards the end of the MIPS, as the active force is lowered, is correlated with the gradual lost of the global hexatic order at the inner part of the dense slab. The authors of Ref. 34] have recently demonstrated, for a minimum continuum active model, how a negative capillary tension may result into a microphase-separated state or into an active foam state, rather than the MIPS of a large dense cluster.

We propose to make use of the approach presented in Ref. 52], based on a density functional description for capillary wave fluctuations on free liquid surfaces. The reason behind this choice is that this approach can be safely applied to non-equilibrium systems. CWT can be applied to a liquid or a solid surface, as long as the system is kept above the roughening temperature. Our system satisfies this condition, being two dimensional and active. 
Moreover, choosing to study the surface fluctuations at small wavevectors guarantees to safely apply CWT, given the low upper-cutoff wavevector typical of a solid surface.

Thus, we estimate the surface tension in a phase separated system of Active Brownian Particles. We unravel the relevance of inter-particle interactions (whether repulsive or attractive) in both stability and structural features of the dense MIPS phase. We compare the results for ABP with WCA-LJ repulsive interactions with those including an attractive range $\left(r_{c}=2.5 \sigma\right)$ from the tail of the LJ potential. Over the range of high activity strength that produces MIPS we find no qualitative difference and very little quantitative effects of that attractive interaction, that becomes crucial for much lower strength of the active force when a equilibrium-like condensation phase transition may appear.

\section{NUMERICAL AND THEORETICAL DETAILS}

\section{A. Model and Numerical details}

A suspension of active Brownian particles (ABP) consists of particles undergoing a Brownian motion with an additional constant self-propelling force $F_{a}$ acting along their orientation vector $\vec{n}=\left(\cos \theta_{i}, \sin \theta_{i}\right)$, that fluctuates independently for each particle. The equations of motion are

$$
\begin{gathered}
\dot{\vec{r}}_{i}=\frac{D}{k_{B} T}\left(-\sum_{j \neq i} \nabla V\left(r_{i j}\right)+\left|F_{a}\right| \vec{n}_{i}\right)+\sqrt{2 D} \vec{\xi}_{i}, \\
\dot{\theta}_{i}=\sqrt{2 D_{r}} \eta_{i} .
\end{gathered}
$$

being $\eta_{i}$ and the components of $\vec{\xi}_{i}=\left(\xi_{i}^{(1)}(t), \xi_{i}^{(2)}(t)\right)$ a white stochastic noise with zero mean and correlations $\left\langle\eta_{i}(t) \eta_{j}\left(t^{\prime}\right)\right\rangle=\left\langle\xi_{i}^{(1)}(t) \xi_{j}^{(1)}\left(t^{\prime}\right)\right\rangle=\left\langle\xi_{i}^{(2)}(t) \xi_{j}^{(2)}\left(t^{\prime}\right)\right\rangle=$ $\delta_{i j} \delta\left(t-t^{\prime}\right) . \quad V\left(r_{i j}\right)$ is the inter-particle pair potential: to study a suspension of repulsive ABP, we consider a WCA, i.e. a LJ truncated and shifted at its minimum $r_{c}=2^{1 / 6} \sigma$; whereas for a suspension of attractive ABP, we consider a LJ truncated and shifted at $r_{c}=2.5 \sigma$, that includes attractive interactions. $\sigma=1$ and $\epsilon=1$ are units of length and energy. The temperature $T$, in units of the Boltzmann constant $k_{B}$ and $\epsilon$, is set at $k_{B} T / \epsilon=0.42$. We use the translational diffusion constant $D=1$ to set the time scale, $\tau=\sigma^{2} / D$. The rotational diffusion is set to obey the Stokes-Einstein equations: $D_{r}=3 D / \sigma^{2}$.

We carry out simulations of a two dimensional suspension of $\mathrm{ABP}$ with an in house modified version of the LAMMPS [53, 54] open source package. The system consists of $N=40000$ particles in a two-dimensional box with periodic boundary conditions, that may be kept with fixed $(N, V, T)$ or fluctuating $(N, p, T)$ to stabilize and analyse a MIPS slab (see Supplementary Info for more details). The time step has been set to $\Delta t=10^{-5} \tau$.

As a measure of the degree of activity, we define the dimensionless $F_{a} \sigma / \epsilon$, i.e. the constant active force modulus $F_{a}$ in units of the LJ parameters [55]. Having to compare repulsive versus attractive particles, for large values of $F_{a}$, and for the structural properties explored here, the value of the LJ-scaled $F_{a}$ is more relevant that the thermal Péclet number $\mathrm{Pe}=\frac{3 v}{\sigma D_{\mathrm{F}}}$, where $v=\left|F_{a}\right| D / k_{B} T$ is the self-propelling velocity $[1]$. Using the above mentioned parameters, we can relate the Péclet number $\mathrm{Pe}=\frac{3 v}{\sigma D_{r}}$ to the Peclet obtained by means of our parameter choice: $\mathrm{Pe}=\frac{3 v}{\sigma D_{r}}=\frac{F_{a} \sigma}{\epsilon} \frac{1}{0.42}$.

\section{B. Theoretical details}

The Capillary Wave Theory 52] (CWT) describes the fluctuations of an interface with surface tension $\gamma_{o}$ through an intrinsic density profile (IDP) $\rho_{I}(x)$ that follows the instantaneous shape of an intrinsic surface (IS). In our 2D system the interface is really a line $x=\xi(y)=\sum_{q} \hat{\xi}_{q} e^{i q y}$ (considering the $Y$ axis along the dense slab), but we keep the trend of previous authors and use "intrinsic surface" and "surface tension", instead of "intrinsic line" and "line tension". Nevertheless, the $1 \mathrm{D}$ dimensional character of the interface is reflected in an enhanced dependence of the mean density profile $\rho(x)$ with the size of the system.

The CWT describes that effect from the average over the fluctuations of the IS Fourier components $\hat{\xi}_{q}: \rho(x)=$ $\left\langle\rho_{I}(x-\xi(y))\right\rangle_{\xi}$, which in a thermal equilibrium system could be predicted from Bolztmann distribution. In our ABP system we cannot use such thermal equibrium hypothesis, but still we may cast in the CWT terms of the IS and the IDP the results of our simulations.

Considering an ABP suspension undergoing MIPS (Fig. 1), a density gap $\delta \rho_{h l}=\rho_{l}-\rho_{h}$ appears between the low and high density phases. Thus, we use a Gaussian smoothed density distribution $\varrho(x, y)=$ $\frac{1}{2 \pi \alpha} \sum_{i} e^{-\left(\vec{r}-\vec{r}_{i}\right)^{2} /\left(2 \alpha^{2}\right)}$, being $\vec{r}_{i}$ particle positions, to define the IS from the equation $\varrho(\xi(y), y)=\left(\rho_{h}+\rho_{l}\right) / 2$, for each $y$, and to get the Fourier components $\hat{\xi}_{q}$ for the wavevectors allowed by the cell size $L_{y}$, up to an upper cutoff $|q| \leq q_{u}$. Fig. 1 represents a typical MIPS configuration (bottom panel, for an attractive ABP system) together with a density profile (top panel). The snapshot in the bottom panel illustrates ABP (in green) and the $x=\xi(y)$ lines on both sides of the dense phase boundaries (in black), calculated with our choice $\alpha=2 \sigma$ and $q_{u}=0.5 / \sigma$. To estimate the resolution of our mesoscopic description, we report in the two insets the $x=\xi(y)$ lines for a different choice of $\alpha$ and $q_{u}$. The top panel in Fig. 1 shows the mean density profile $\rho(z)=\left\langle\sum_{i} \delta\left(x-x_{i}\right)\right\rangle / L_{y}$ that averages the position of the particles over 2000 configurations separated by 100000 steps.

As suggested by Ref. [3], in an ABP-MIPS system, 


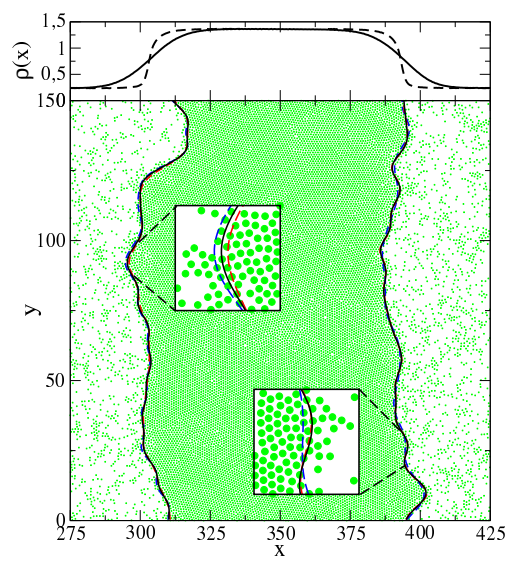

FIG. 1. Snapshot of the attractive ABP system, characterised by $F_{a}=55$. The IS is drawn at the two edges of the dense slab, for different choices of the parameters. The black lines (on both sides) correspond to our preferred choice, $\alpha=2$ for the Gaussian coarse-grain and $q_{u}=0.5$ as the upper cutoff in the Fourier series. On the left side-inset, the dashed lines show the effect of changing to $\alpha=1$ (red) and $\alpha=3$ (blue), keeping $q_{u}=0.5$. On the right side-inset, we keep $\alpha=2$ and change to $q_{u}=0.25$ (red) and $q_{u}=1.5$ (blue). Top panel: mean density profile $\rho(x)$ (continuous line); intrinsic density profiles $\rho_{\mathrm{I}}(x)$ (dashed line), with their origin set at the mean position of each edge.

despite the random direction $\vec{n}_{i}$ of the active force on each particle, the mean active force profile $\vec{f}^{a}(x)=$ $F_{a}\left\langle\sum_{i} \vec{n}_{i} \delta\left(x-x_{i}\right)\right\rangle / L_{y}$ does not vanish at the interface. Therefore, as for the IDP, we may get the intrinsic active force profiles $\vec{f}_{\mathrm{I}}^{a}(x)=\left|F_{a}\right|\left\langle\sum_{i} \vec{n}_{i} \delta\left(x-x_{i}+\xi\left(y_{i}\right)\right\rangle / L_{y}\right.$, for a sharper view of the force distribution at the interface. In steady state, the mean active force has to be compensated by the mean repulsion between particles (which may be used as a test for statistics in the ABP simulations, see Supplementary Info). Since interfaces are along the $Y$ direction, only the $X$ component of the mean active force is not null: we refer to it in all the following as $f_{\mathrm{I}}^{\mathrm{a}}(x)$.

The intrinsic profiles may be computed according to particles with a given coordination $\nu$, i.e. the number of neighbors within repulsion distance $\left(r_{i j} \leq 1.12 \sigma\right)$. Most of the particles in the dense slab have $\nu=6$, with triangular lattice coordination, measured by the local hexatic order parameter, $\eta_{j}=\sum_{k} \exp \left(6 i \theta_{k}\right) / 6$, where $\theta_{k}$ is the orientation of the relative position $\vec{r}_{j k}$ for the $(k=1$ to 6 ) neighbors of particle $j$. For each particle in the dense phase, this parameter has a modulus $\left|\eta_{j}\right| \approx 1$, but its complex phase reflects the local orientation of the neighbour. The global $\eta=\left\langle\sum_{i} \eta_{i}\right\rangle / N$, averaged over $N \gg 1$ particles, may have a modulus well below 1 , because of the different phase of each $\eta_{i}$. The hexatic 2D phase ap- pears when $|\eta|$ is well above the noise $\left(\sim N^{-1 / 2}\right)$. In our ABP simulations we have calculated $\eta$ over the central half of the dense slab and, as it had been described before [17, 56 58], near the borders there are large grains, with different phases of their $\eta$ averages. The global hexatic order is gradually lost by accumulation of $\nu=5$ and 7 defects in a mesh of disclination lines denser at the edges.

Besides the intrinsic profiles, the CWT analysis gives information on the IS fluctuations. As for equilibrium fluid interfaces [52], we get independent Gaussian distributions for the Fourier components $\hat{\xi}_{q}$ (S.I.) and the mean square amplitudes, written as $\left\langle\left|\hat{\xi}_{q}\right|^{2}\right\rangle=\left(q^{2} \beta \gamma(q) L_{y}\right)^{-1}$, define a wavevector dependent surface (or line) tension $\gamma(q)$. In equilibrium systems these mean square fluctuations are proportional to $k T=\beta^{-1}$, and $\gamma(q)$ gives the macroscopic surface tension as the limit $\gamma_{o}=\gamma(0)$. In $\mathrm{ABP}$ interfaces we have to interpret $\beta \gamma(q)$ together, as an (inverse) measure of the IS fluctuations.

\section{RESULTS AND DISCUSSION}

Besides having prepared the initial configuration for a suspension of repulsive (WCA) ABPs, we study the effect of activity on a phase separated system of attractive (LJ) active particles. We prepare a phase separated system of attractive particles at thermodynamic conditions at which its passive counterpart would phase separate. In Figure 2 panel a) we observe that adding a small activity to the attractive particles is not enough to break the dense-dilute phase separation (also detected for the equilibrium passive system): for small values of activity $\left(F_{a}<3\right)$ the dense phase is stable as a band.

a)

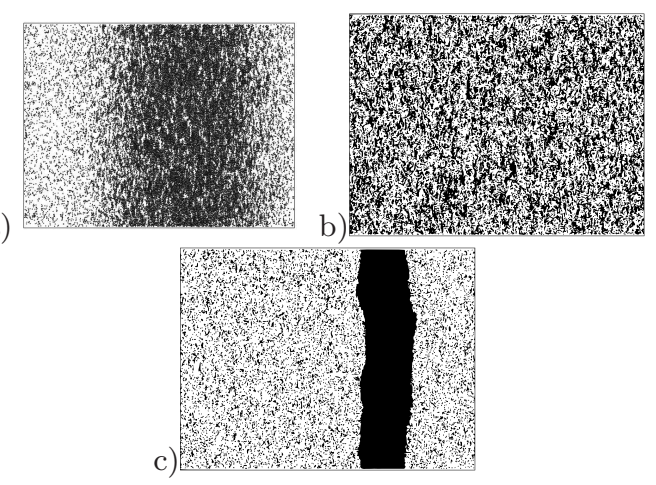

FIG. 2. Snapshots of an attractive LJ-ABP suspension with $F_{a}=\{3,30,80\}$ respectively a,b,c.

Similarly to previous studies [6], we observe a reentrant phase behavior of the phase separated structure. When increasing the propulsion strength, $3<F_{a}<50$, (Figure 2 panel a to b), activity overtakes attraction and the band melts, leading to a gas phase. Strikingly, if we increase propulsion strength even further $\left(F_{a}>40\right)$, phase coexistence emerges again (Figure2 2 panel c). The system 
forms a more compact band, even though the mean density in the box is quite low $\rho=0.38$. In what follows, we will consider either repulsively or attractively interacting particles at relatively high activity, where attraction is less relevant than propulsion.

Having prepared the initial configuration, we estimate the mean density profiles and the IDP for a dense-dilute MIPS formed by both repulsive and attractive ABP. Figure3 presents the IDPs for three values of activity. To eliminate the $F_{a}$ dependence of the bulk densities, we scale it and shift it $\left(2 \rho_{\mathrm{I}}(x)-\rho_{h}-\rho_{l}\right) / \Delta \rho_{h l}$.

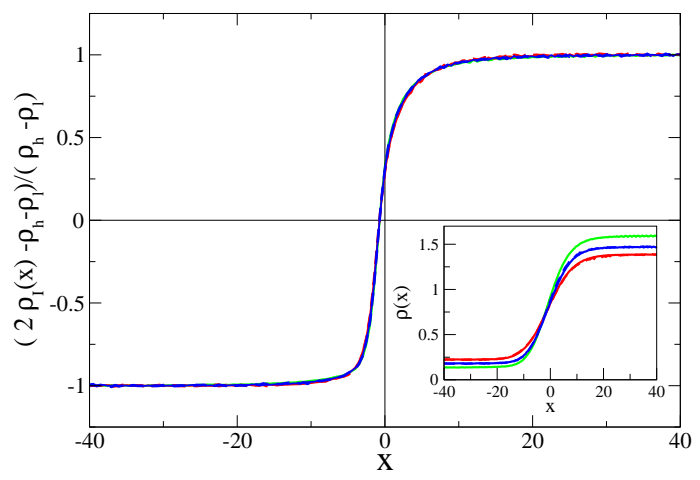

FIG. 3. (Mean (inset) and intrinsic (main panel) density profiles for $F_{a}=60$ (red), 75 (blue) and 120 (green). The IDP are scaled and shifted to get \pm 1 as the limit values at the two sides of the interface, and the results for all the $F_{a}$ values become nearly identical. Although indistinguishable, the full lines are the LJ-ABP results and the dashed line the WCAABP. Units of the LJ $\sigma$ diameter are used.

All scaled IPDs become nearly identical and are asymmetric, with a smoother decay towards the dense side. The inset in Fig 3 shows the mean density profiles that become broader as we reduce $F_{a}$. The mean density profiles $\rho(x)$ become smoother for decreasing activity because of the larger IS fluctuations, rather than from any local change in the interfacial region, other than the scaling of the bulk densities. Interestingly, the results obtained for repulsive particles coincide with the ones for attractive ones. For the chosen activity range, the interparticle interaction plays no role in the mean density profile.

To better characterise the interface, we study the density and the active force profiles for different coordination numbers, when the dense-dilute MIPS is made of

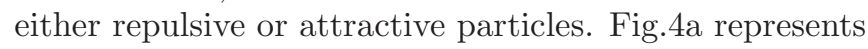
mean density profiles $\rho(x)$ (continuous lines) and intrinsic density profiles $\rho_{I}(x)$ (dashed lines) for coordination numbers $\nu \leq 3$ (green lines), $\nu=4$ (blue lines), $\nu=5$ or $\nu=7$ (red lines) and $\nu=6$ (magenta lines).

In the low density region, comparing the coloured lines, we conclude that the low density phase is mostly made of particles with $\nu \leq 3$ (green line). Moreover, the rapid increase of $\rho_{\mathrm{I}}(x)$ (dashed line) from the $\rho_{l}$ bulk, comes from the accumulation of $\nu=2-3$ particles at the edge of the dense slab. Particles with $\nu=4$ (blue line) are
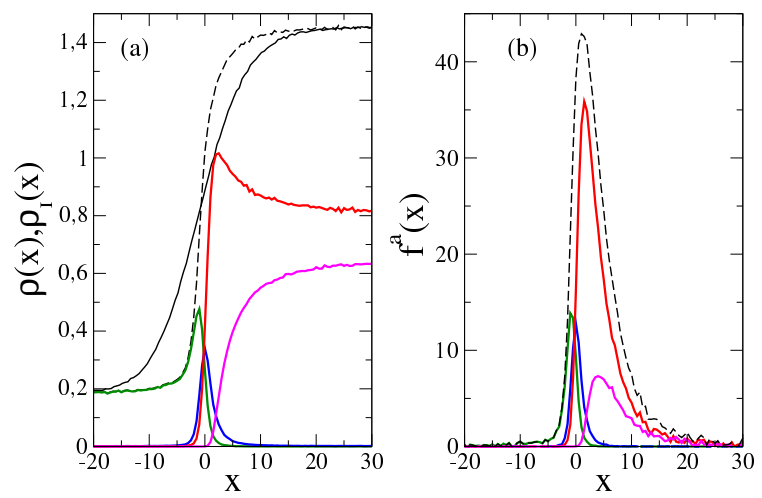

FIG. 4. Density (panel a) and active force (panel b) profiles for the LJ-ABP with $F_{a}=80$. Full black line (a) mean density profiles. The intrinsic profiles (dashed black lines in both panel) are split in particles with $\nu \leq 3$ (green), $\nu=4$ (blue), $\nu=5$ or 7 (red), and $\nu=6$ (magenta) coordination numbers.

distributed as a very narrow peak at $x=\xi(y)$, which provides an independent test for our IS definition. In the high density phase we get $\nu \geq 5$ (red and magenta lines) and the very slow raise of $\rho_{\mathrm{I}}(x)$ is produced mainly by the gradual decay of five-fold correlated particles, as we move into the bulk.

Fig $4 \mathrm{~b})$ represents the active force profiles $f_{a}(x)$ for the mean density (continuous lines) and for the intrinsic density (dashed lines), and for coordination numbers $\nu \leq 3$ (green lines), $\nu=4$ (blue lines), $\nu=5$ or $\nu=7$ (red lines) and $\nu=6$ (magenta lines). When $\nu \leq 4$, the profile is peaked at the low density region, whereas when $\nu \leq 5$ the peak becomes much higher and moves towards the high density region. The high density slab is compressed to a higher pressure than the lower density phase, which make the MIPS interface to be mechanically very different from an equilibrium liquid-vapor surface. Notice this interpretation in terms of different pressure between coexisting phases is that of Ref. [3],, while other authors used to include the effect of the active force as part of the stress tensor, rather than as an effective external force, and get equal pressure between coexisting phases. Both approaches are equally valid, if treated consistently.

The active force profiles demonstrate how the correlation with the particle position rectifies the random orientation of the active force and creates the effect of a mean external potential at the interface [3]. The rectification of the active force was interpreted as the result of the swimming pressure of the low density phase on the edge of the dense slab. When the active force on these highly mobile particles points inward $\left(n_{x}>0\right)$ it is arrested by the repulsion of the particles in the slab, but when $n_{x}<0$ the particles are free to move out of the interface, they dissipate the active force as friction, and become scarcer at the interface. 
Our intrinsic view for the active force profiles allows a much sharper characterization and gives a different perspective. The rectification of the active force comes mainly from the denser side of the interface, rather than from the external layer of freely moving particles. Given that mobility in the dense phase is small, the local active force's rectification happens on time scales longer than the typical characteristic times of the angular diffusion. The $x$ component of the active force has an asymmetric peak extended over distances $-5 \sigma \leq x \leq 20 \sigma$ from the IS, similar to the asymmetry of the intrinsic density $\rho_{\mathrm{I}}(x)$. The splitting of $f_{\mathrm{I}}^{\mathrm{a}}(x)$ in terms of $\nu$ shows that the rectification of the random active force comes mainly from particles with crystalline correlation $(\nu=6)$ or at lattice point defects $(\nu=5,7)$. Apparently, these particles should be too tightly caged by their neighbors to get the asymmetric mobility that rectifies their active force as a swimming pressure.

The explanation for this feature comes from the slow raise of $\rho_{I}(x)$ towards the dense phase, which signals the accumulation of point defects (mostly particles with $\nu=5$ coordination) over that thick region $\sim 20 \sigma$ at the edge of the slab. Such accumulation of lattice defects implies the presence of disclination lines, or hexatic grain boundaries, that gradually break the nearly perfect $2 \mathrm{D}$ crystal correlation in the interior of the dense slab when we approach the interface [17, 56? -59]. The mobility of particles along these grain boundaries should be asymmetric, more easily (collectively) shifted by the active force when $\vec{n}_{i}$ points towards the interface than when it pushes towards the interior of the slab.

The gradual increase of the compression, as we move towards the center of the slab, reduces the defects, slowly increasing the density $\rho_{\mathrm{I}}(x)$, and that reduces the rectification of the active force $F_{a} \vec{n}$. At the inner part of the slab the distribution of lattice defects and dislocation lines becomes statistically homogeneous, the rectification of the active force disappears, and (consistently) the central part of the dense slab becomes a homogeneous hexatic phase characterised by bulk density.

Finally, within the CWT analysis we compute the mean square amplitude of the IS fluctuations, through the function $\beta \gamma(q)=\left(q^{2}\left\langle\left|\hat{\xi}_{q}\right|^{2}\right\rangle L_{y}\right)^{-1}$, as shown in Fig. 5 (top panel).

To study the limit of $\beta \gamma(q)$ at low $q$, we use the following fit $\beta \gamma(q) \approx \beta \gamma_{o}+\beta \kappa q^{2}$, that is quite accurate and gives robust results (Fig. 5 inset). Figure 5 - top panel presents $\beta \gamma_{o}$ as a function of $F_{a}$. The error bars have been computed to give the difference between the results obtained on the two edges of the slab.

In a thermal equilibrium liquid-vapor interface, the $\beta$ factor may be removed to get (from the analysis of the surface fluctuations) the same $\gamma_{o}$ that gives the mechanical work (the free energy change) for increasing the area. In an ABP MIPS, the mechanical evaluation of $\gamma_{o}$ has led to surprisingly negative values [2]. The interpretation of the MIPS as coexistence of two phases with a bulk pressure difference, self-maintained by the rectification of the

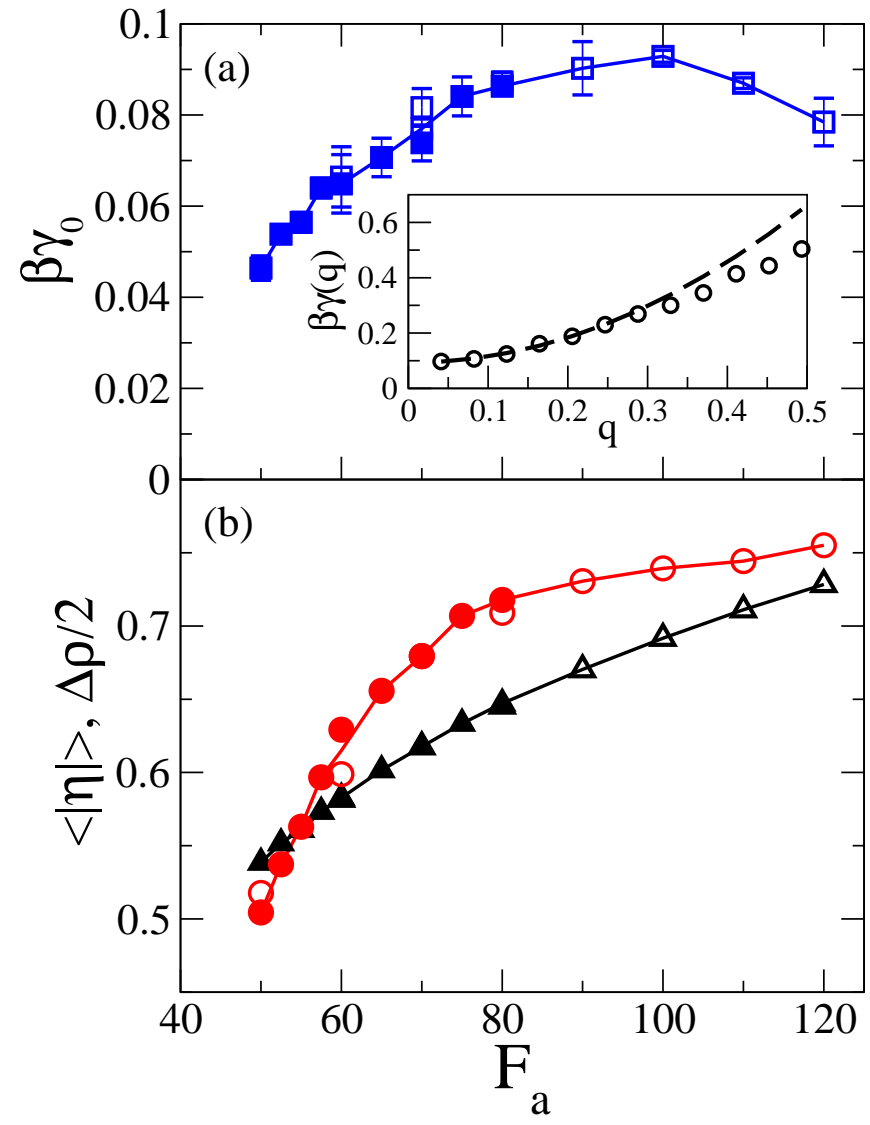

FIG. 5. (a) The value of $\beta \gamma_{o}$ extracted from the mean fluctuations at the two edges of the slab (error bars give the difference between the two sides). (Inset) The function $\beta \gamma(q)$, for the wavevectors $q$ in our simulation cell, for $F_{a}=100$, with its quadratic fit to $\beta \gamma_{o}+\beta K q^{2}$, to extract $\beta \gamma_{0}$ represented in the blue symbols. (b) Half the density gap, $\left(\rho_{h}-\rho_{l}\right) / 2$ (black triangles), and the mean modulus of the hexatic order parameter $\langle|\eta|\rangle$ calculated over the central part of the dense slab (red circle), as functions of the active force $F_{a}$. The solids symbols are the LJ-ABP results and the empty symbols the WCA-ABP, the lines are eyes guide.

active force at the interfaces [3], voids that controversy. Even if we could get rid of the $\beta$ factor, our $\gamma_{o}$ could not be used to get the mechanical work of a global deformation of the system without including the pressure difference between the two phases. Our capillary surface tension, $\beta \gamma_{o}$, is always positive, and it would vanish only if the interfacial fluctuations become unbounded and destroy the MIPS.

To conclude, we study how MIPS disappears for $F_{a} \leq$ 50. The usual description is presented in terms of the density gap $\Delta \rho_{h l}$ in the $\left(\rho, F_{a}\right)$ phase diagrams [17], as for equilibrium phase transitions. Our analysis adds two clearer clues with the rapid decay of the hexatic order parameter $\langle|\eta|\rangle$ over the bulk of the dense phase, and of $\beta \gamma_{o}$ at the interfaces. Figure 5 bottom panel reports half the density gap, $\left(\rho_{h}-\rho_{l}\right) / 2$ and the mean modulus of the hexatic order parameter $\langle|\eta|\rangle$ calculated over the 
central part of the dense slab, as functions of the active force $F_{a}\left(50 \leq F_{a} \leq 120\right)$. The dependence of $\Delta \rho_{h l}$ over that range is very smooth, without any signal that MIPS may be close to its end for $F_{a} \lesssim 50$. However, both $\langle|\eta|\rangle$ and $\beta \gamma_{o}$ show a clear slope change at $F_{a} \approx 80$ and decay rapidly for lower activity.

The connection between these two magnitudes may be rationalized when we interpret the MIPS of the ABP in the terms suggested by the intrinsic density and force profiles. A weaker active force reduces the pressure on the dense slab, and its density decreases mainly by changing the mean nearest neighbor distance. When that effect is scaled out, as in Fig. 3 we get very similar IDP for all the $F_{a}$. However, the hexatic order at the inner part of the slab is rapidly weakened as the density decreases. Large local fluctuations in the hexatic order at the center of the slab would go against the rectification of the random active force, since the disclination lines could reach the inner part of the slab, instead of being mainly restricted to the grain boundaries over the width $\sim 20 \sigma$ at the edges of the slab where $f^{a}(x)$ has its main contribution. The intuitive image that appears from our analysis is that when the active force, rectified through the asymmetric mobility of the particles along the grain boundaries, becomes too weak to keep the inner hexatic order of the dense slab, the grain boundaries may percolate from side to side of the slab and act as wedges that break the dense phase in pieces, to end with the MIPS.

\section{CONCLUSIONS}

Most of the theoretical concepts and tools we have implemented in our work have been recently used by different authors. Patch et al. 32] presented an intrinsic surface analysis of the ABP interface similar to ours; the full phase diagram, including the analysis of the hexatic order, had been studied by several groups 17, 56? 59], and the interpretation of MIPS as an effect of an effective external potential, created by the active force across density gradient, was proposed by Omar et al. [3]. Our contribution relies on unifying all these concepts, together with adding a novel view of the mechanism for MIPS coming from our CWT analysis.

The force generated at the interface, as a rectification of the random direction of active force, comes mainly from the dense side of the interface, through a rather thick layer (of about 20 particle diameters). This is shown in the intrinsic force and density profiles. The latter with an amazing similarity in their scaled shapes over the whole range of MIPS. The image of the swimming pressure, created by nearly free particles in the low density phase under the effects of the random active force, breaks down for the tightly caged particles that contribute most to the compression of the dense phase slab. The rectification of the random active force, to generate an effective external potential, has to come from collective rearrangements of the caged particles, at the hexatic phase grains which had been recently observed and characterized [17, 56 58] as an important feature of the MIPS in 2D-ABP. There is clear correlation between the rapid loss of the hexatic order at the inner part of that slab; the decrease of $\beta \gamma(0)$ (i.e. the growth of the interfacial fluctuations), and the end of the MIPS at $F_{a} \lesssim 50$.

As far as we may ascertain, the end of the MIPS comes for $F_{a}$ just below 50, when the compression by the active force cannot keep the hexatic order at the inner of the slab. That is correlated with the decay of $\beta \gamma_{o}$, i.e. the growth of the geometrical fluctuations of the interface. In their dependence with active force, both the hexatic order parameter $\langle|\eta|\rangle$ and $\beta \gamma_{o}$ show a change at $F_{a} \approx 80$, that is associated to the pinning of the hexatic phase by the direction of the boundaries. Although with different interactions and parameters, this agrees qualitatively with the distinction between "hexatic" and "solid" for the structure of the dense coexisting phase in the full phase diagram [17]. Nevertheless, the accurate characterization of that region of the ABP phase diagram faces great difficulties, because large spatial and temporal scales are needed to simulate the $2 \mathrm{D}$ hexatic crystal slab, with a thick premelted film at each border, and approaching its bulk melting conditions. In this respect, the recent work by Caporusso et al. [56] for the nucleation and growth of the hexatic grains in large ABP simulations indicates that the macroscopic separation of an inner homogeneous dense phase may be strongly propitiated, and perhaps created, by its percolation as a slab, through the periodic boundary conditions.

Finally, at the high $F_{a}$ values explored here, our use of the attractive interaction between particles coincide with results obtained for repulsive particles. However, at much lower $F_{a}$, the system of attractive particles shows a liquid-vapor coexistence, that smoothly goes to the thermal equilibrium transition at $F_{a}=0$. The application of CWT-based analysis methods to those weakly-active LJ systems may be as useful as it has been in the stronglyactive case studied here.

\section{CONFLICTS OF INTEREST}

There are no conflicts to declare.

\section{ACKNOWLEDGEMENTS}

We acknowledge the support of the Spanish Secretariat for Research, Development and Innovation (Grants No. FIS2017-86007-C3, PID2020-117080RBC52, FPU2015/0248, and PID2019-105343GB-I00) and from the Maria de Maeztu Programme for Units of Excellence in R\&D (CEX2018-000805-M). 
[1] J. Stenhammar, D. Marenduzzo, R. Allen, and C. M.E., Soft Matter 10, 1489 (2014).

[2] J. Julian Bialke, J. T. Siebert, H. Lowen, and T. Speck, Phys. Rev. lett. 115, 098301 (2015).

[3] A. K. Omar, Z.-G. Wang, and J. F. Brady, Phys. Rev. E 101, 012604 (2020).

[4] C. Bechinger, R. Di Leonardo, H. Lowen, C. Reichhardt, G. Volpe, and G. Volpe, Reviews of Modern Physics. 88, 045006 (2016).

[5] G. S. Redner, M. F. Hagan, , and A. Baskaran, Physical Review Letters 110, 055701 (2013).

[6] G. S. Redner, A. Baskaran, and M. F. Hagan, Phys. Rev. E 88, 012305 (2013)

[7] B. M. Mognetti, A. Saric, S. Angioletti-Uberti, A. Cacciuto, C. Valeriani, and D. Frenkel, Physical Review Letters 111, 245702 (2013).

[8] F. Alarcon, C. Valeriani, and I. Pagonabarraga, Soft matter 13, 814 (2017).

[9] D. Sarkar, G. Gompper, and J. Elgeti, Commun. Phys. 4, 36 (2021).

[10] J. Palacci, S. Sacanna, A. P. Steinberg, D. J. Pine, and P. M. Chaikin, Science 339, 936 (2013).

[11] I. Buttinoni, J. Bialke, F. Kummel, H. Lowen, C. Bechinger, and T. Speck, Physical review letters 110, 238301 (2013).

[12] I. Theurkauff, C. Cottin-Bizonne, J. Palacci, C. Ybert, and L. Bocquet, Physical Review Letters 108, 268303 (2012).

[13] S. S. Hermann, D. de las Heras, and M. Schmidt, Mol. Phys. 119, e1902585 (2021).

[14] S. Thutupalli, R. Seemann, and S. Herminghaus, New Journal of Physics 13, 073021 (2011).

[15] D. D. Nishiguchi and M. Sano, Physical Review E 92, 052309 (2015)

[16] G. Briand and O. Dauchot, Phys. Rev. Lett. 117, 098004 (2016).

[17] P. Digregorio, D. Levis, A. Suma, L. F. Cugliandolo, G. Gonnella, and I. Pagonabarraga, Phys. Rev. Lett. 121, 098003 (2018).

[18] J. Martin-Roca, R. Martinez, L. C. Alexander, A. L. Diez, D. G. A. L. Aarts, F. Alarcon, J. Ramírez, and C. Valeriani, The Journal of Chemical Physics 154, 164901 (2021)

[19] A. P. Solon, J. Stenhammar, M. E. Cates, Y. Kafri, , and J. Tailleur, New J. Phys. . 20, 075001 (2018).

[20] L. Caprini, E. Hernandez-Garcia, C. Lopez, and U. Marini Bettolo Marconi, Scientific Reports 9, 16687 (2019).

[21] D. Levis, J. Codina, and I. Pagonabarraga, Soft Matter 13, 8113 (2017).

[22] X.-q. Shi, G. Fausti, H. Chate, C. Nardini, and A. Solon, Physical Review Letters 125, 168001 (2019).

[23] A. Wysocki, R. Winkler, and G. Gompper, EPL 105, 48004 (2014).

[24] H. Lowen, J. Chem. Phys. 152, 040901 (2020).

[25] J. Su, H. Jiang, and Z. Hou, New J. Phys. 23, 013005 (2021).

[26] F. Dittrich, T. Speck, and P. Virnau, Eur. Phys. J. E 44, 53 (2021).

[27] C. M. Barriuso, C. Vanille, F. Alarcon, I. Pagonabarraga, R. Brito, and C. Valeriani, "Collec- tive motion of run-and-tumble repulsive and attractive particles in one dimensional systems," (2021), arXiv:1912.01282 [cond-mat.soft]

[28] D. Rogel Rodriguez, F. Alarcon, R. Martinez, J. Ramírez, and C. Valeriani, Soft Matter 16, 1162 (2020)

[29] J. Stenhammar, R. Wittkowski, D. Marenduzzo, , and M. Cates, Phys.Rev.Lett. 114, 018301 (2015).

[30] A. Wysocki, R. Winkler, and G. Gompper, New J. Phys. 18, 123030 (2016).

[31] J. T. Siebert, F. Dittrich, F. Schmid, K. Binder, T. Speck, and P. Virnau, Phys. Rev. E 98, 030601 (2018).

[32] A. Patch, D. M. Sussman, D. Yllanes, and M. C. Marchetti, Soft Matter 14, 7435 (2018)

[33] S. Paliwal, V. Prymidis, L. Filion, and M. Dijkstra, J. Chem. Phys. 147, 084902 (2017).

[34] G. Fausti, E. Tjhung, M. E. Cates, and C. Nardini, Phys. Rev. Lett. 127, 068001 (2021)

[35] G. F, I. Theurkauff, D. Levis, C. Ybert, L. Bocquet, L. Berthier, and C. Cottin-Bizonne, Phys. Rev. X 5, 011004 (2015).

[36] Y. Fily, A. Baskaran, and M. F. Hagan, Soft matter 10, 5609 (2014).

[37] S. C. Takatori, W. Yan, and J. F. Brady, Phys. Rev. Lett. 113, 1 (2014).

[38] S. A. Mallory, A. K. Omar, and J. F. Brady, "Dynamic overlap concentration scale of active colloids," (2020), arXiv:2009.06092 [cond-mat.soft]

[39] J. M. Epstein, K. Klymko, and K. K. Mandadapu, J. Chem. Phys. 150, 1 (2019).

[40] R. G. Winkler, A. Wysocki, and G. Gompper, Soft Matter 11, 6680 (2015).

[41] A. Patch, D. Yllanes, and M. C. Marchetti, Phys. Rev. E 95, 012601 (2017).

[42] A. Solon, Y. Fily, A. Baskaran, M. Cates, Y. Kafri, M. Kardar, and J. Tailleur, Nat. Phys. 11, 673 (2015).

[43] T. Speck and R. Jack, Phys. Rev. E 93, 062605 (2016).

[44] Y. Fily, Y. Kafri, A. Solon, J. Tailleur, and A. Turner, J. Phys. A 51, 044003 (2018).

[45] S. Das, G. Gompper, and R. Winkler, Sci. Rep. 9, 6608 (2019).

[46] A. Trokhymchuk and J. Alejandre, J. Chem. Lett. 111, 8510 (2013).

[47] N. Lauersdorf, T. Kolb, M. Moradi, E. N. E, and D. Klotsa, Soft Matter 17, 6337 (2021).

[48] J. S. Rowlinson and B. Widom, Molecular Theory of Capillarity (Claredon Press, 1982).

[49] F. P. Buff, R. A. Lovett, and F. H. Stillinger, Phys. Rev. Lett. 15, 621 (1965).

[50] E. Chacón and P. Tarazona, Phys. Rev. Lett. 91, 166103 (2003).

[51] R. Delgado-Buscalioni, E. Chacón, and P. Tarazona, Phys. Rev. Lett. 101, 106102 (2008).

[52] P. Tarazona, R. Checa, and E. Chacón, Phys. Rev. Lett. 99, 196101 (2007).

[53] S. Plimpton, J. Comp. Phys. 117, 1 (1995).

[54] D. Rogel Rodriguez, F. Alarcon, R. Martinez, J. Ramírez, and C. Valeriani, Soft Matter 16, 1162 (2020)

[55] Note that this ratio is related to the $\xi$ reported in Ref[8].

[56] C. B. Caporusso, P. Digregorio, D. Levis, L. F. Cugliandolo, and G. Gonnella, Phys. Rev. Lett. 125, 178004 
(2020).

[57] P. Digregorio, D. Levis, L. F. Cugliandolo, G. Gonnella, and I. Pagonabarraga, "Clustering of topological defects in two-dimensional melting of active and passive disks,"
(2021), arXiv:1911.06366 [cond-mat.soft]

[58] S. Paliwal and M.

Phys. Rev. Research 2, 012013 (2020)

[59] J. Klamser, S. K. S.C., and W. Krauth, Phys. Rev. Lett. 9, 5045 (2018).
Dijkstra, 